\title{
Mobilization of intensive care patients: a multidisciplinary practical guide for clinicians
}

This article was published in the following Dove Press journal:

Journal of Multidisciplinary Healthcare

25 May 2016

Number of times this article has been viewed

\section{Margot Green' \\ Vince Marzano' \\ I Anne Leditschke ${ }^{2,3}$ \\ Imogen Mitchell ${ }^{2,3}$ \\ Bernie Bissett ${ }^{1,4,5}$}

'Physiotherapy Department, Canberra Hospital, Canberra, ACT, Australia; ${ }^{2}$ Intensive Care Unit, Canberra Hospital, Canberra, ACT, Australia; ${ }^{3}$ School of Medicine, Australian National University, Canberra, ACT, Australia; ${ }^{4}$ Discipline of Physiotherapy, University of Canberra, Canberra, ACT, Australia; ${ }^{5}$ School of Medicine, University of Queensland, Brisbane, QLD, Australia
Correspondence: Bernie Bissett Physiotherapy Department, Canberra Hospital, Building 15, Yamba Drive, Garran, Canberra, ACT 2605, Australia Tel +6I 262442154

Fax +6I 262443692

Email Bernie.Bissett@act.gov.au
Objectives: To describe our experience and the practical tools we have developed to facilitate early mobilization in the intensive care unit (ICU) as a multidisciplinary team.

Background: Despite the evidence supporting early mobilization for improving outcomes for ICU patients, recent international point-prevalence studies reveal that few patients are mobilized in the ICU. Existing guidelines rarely address the practical issues faced by multidisciplinary ICU teams attempting to translate evidence into practice. We present a comprehensive strategy for safe mobilization utilized in our ICU, incorporating the combined skills of medical, nursing, and physiotherapy staff to achieve safe outcomes and establish a culture which prioritizes this intervention.

Methods: A raft of tools and strategies are described to facilitate mobilization in ICU by the multidisciplinary team. Patients without safe unsupported sitting balance and without $\geq 3 / 5$ (Oxford scale) strength in the lower limbs commence phase 1 mobilization, including training of sitting balance and use of the tilt table. Phase 2 mobilization involves supported or active weight-bearing, incorporating gait harnesses if necessary. The Plan B mnemonic guides safe multidisciplinary mobilization of invasively ventilated patients and emphasizes the importance of a clearly articulated plan in delivering this valuable treatment as a team.

Discussion: These tools have been used over the past 5 years in a tertiary ICU with a very low incidence of adverse outcomes $(<2 \%)$. The tools and strategies described are useful not only to guide practical implementation of early mobilization, but also in the creation of a unit culture where ICU staff prioritize early mobilization and collaborate daily to provide the best possible care. Conclusion: These practical tools allow ICU clinicians to safely and effectively implement early mobilization in critically ill patients. A genuinely multidisciplinary approach to safe mobilization in ICU is key to its success in the long term.

Keywords: physiotherapy (techniques), critical care, intensive care, multidisciplinary communication

\section{Introduction}

Early progressive mobilization of adult intensive care unit (ICU) patients has been shown to be safe and feasible and ${ }^{1,2}$ result in reduced delirium, ${ }^{3}$ improved functional outcomes, ${ }^{4,5}$ reduced hospital length of stay, ${ }^{3,6}$ and reduced mortality in patients with acute respiratory failure. ${ }^{7}$ In a recent systematic review of physiotherapy in ICU, Stiller ${ }^{6}$ suggested that, given the evidence supporting the outcomes for early mobilization, "ICU physiotherapists should give priority to interventions aimed at early progressive mobilization". However, point-prevalence studies from Germany ${ }^{8}$ and Australia and 
New Zealand ${ }^{9}$ have demonstrated a low incidence of mobilization of patients with an endotracheal tube ( $8 \%$ in Germany and $0 \%$ in Australia and New Zealand).

Although there are several guidelines available that discuss the implementation of early mobilization in critically ill patients, ${ }^{10-13}$ few papers address the practical challenges faced by clinicians attempting to translate this evidence into practice. Our 31-bed mixed tertiary ICU had 1,976 admissions in 2015, with 50\% requiring mechanical ventilation. Patients managed include medical, trauma, cardiothoracic, general, and neurosurgical patients. We have been practicing early mobilization and rehabilitation for over a decade, and it is well embedded in our unit culture across the multidisciplinary team (MDT) (physiotherapy, medical, nursing, and support staff $\left.{ }^{14}\right)$. In this paper, we share the practical tools we have developed to educate and train staff in early mobilization across the spectrum of acuity, including patients with neurological diagnoses. Our approach is applicable to general ICU patients regardless of whether they are intubated and mechanically ventilated, noninvasively ventilated, or not requiring any ventilatory or airway support.

Prior to any mobilization episode, a comprehensive assessment and review of safety criteria should occur to minimize risk. This paper is intended to be used in conjunction with published expert consensus statements ${ }^{13,15-17}$ and recommendations and is focused more on the practical implementation of mobilization once a safety assessment has been completed in collaboration with the MDT.

\section{Mobilization methods}

We perform a daily assessment of all patients in the ICU regarding their suitability for mobilization with the aim of achieving the highest level of mobilization possible each day. To determine the type of mobilization, a stepwise approach is taken (Figure 1). The first two steps are to assess the patient's level of alertness and ability to follow instructions (eg, using the Richmond Agitation-Sedation Scale) ${ }^{18}$ and identify if there are any other safety concerns or barriers to mobilization. The physiotherapist will discuss any safety concerns with senior medical staff to determine whether these factors should preclude mobilization. In particular, the team must also consider whether the patient has sufficient respiratory and cardiovascular reserve to perform the proposed mobilization task and if acceptable limits of organ support can be established to facilitate mobilization (eg, alteration of ventilator settings or increasing vasoactive infusions). If sedation can be weaned or barriers to mobilization overcome (eg, timing of procedures or tests), this is prioritized by the MDT early in the day to facilitate mobilization. If any member of the MDT has concerns about whether mobilization should proceed, this is discussed openly with the ICU physiotherapist and senior medical staff.

The flowchart in Figure 1 also reinforces the need to continuously monitor the patient during mobilization. For each patient, we clearly articulate and agree on target ranges for physiological parameters during mobilization, rather than arbitrary thresholds. It is important to remember that the normal physiological response to exercise includes an increase in heart rate and to allow for this when setting parameters. Mobilization may need to temporarily cease due to the patient's physiological response to mobilization (eg, decrease in $\mathrm{SpO}_{2}$ and excessive increase in heart rate). However, after a period of monitoring and rest, mobilization may recommence. In the very rare circumstance of an adverse event, ${ }^{14}$ mobilization ceases immediately and a referral is made for an urgent medical review.

Ultimately, treatment dosage and intensity should align with the specific goals of treatment for the individual patient, whether this is enhanced respiratory status, maintenance of global function or recovery of strength, endurance or balance deficits, or a combination of these. These goals are collaboratively determined by the physiotherapist, bedside nurse, and patient following assessment. Where patients are able to rate their perceived exertion, ${ }^{19}$ this can be helpful in guiding treatment intensity and has shown to be reliable in mechanically ventilated patients. ${ }^{20}$ For example, we may encourage a patient to work at a rated perceived exertion of 3-4 out of 10 while mobilizing around the ICU and reassure them that it will feel challenging at this point in their recovery.

\section{Bed rest}

Patients who are awake but must remain on bed rest due to the presence of safety criteria are assessed for their suitability to complete an in-bed exercise program. This program is individually prescribed to maintain strength and/or range of motion. Physiotherapists liaise closely with surgical and medical teams to clarify movement restrictions and duration of bed rest (eg, following pelvic fracture) to determine when active mobilization can commence.

\section{Passive mobilization}

If the patient is unable to follow commands and actively participate in mobilization (eg, Richmond Agitation-Sedation Score $<-1),{ }^{18}$ he or she may be suitable for passive mobilization (ie, hoist transfer to sit-out-of-bed, Figure 2). Even patients who are sedated and unresponsive may still benefit from the high 
A

Physiotherapy mobilization flowchart

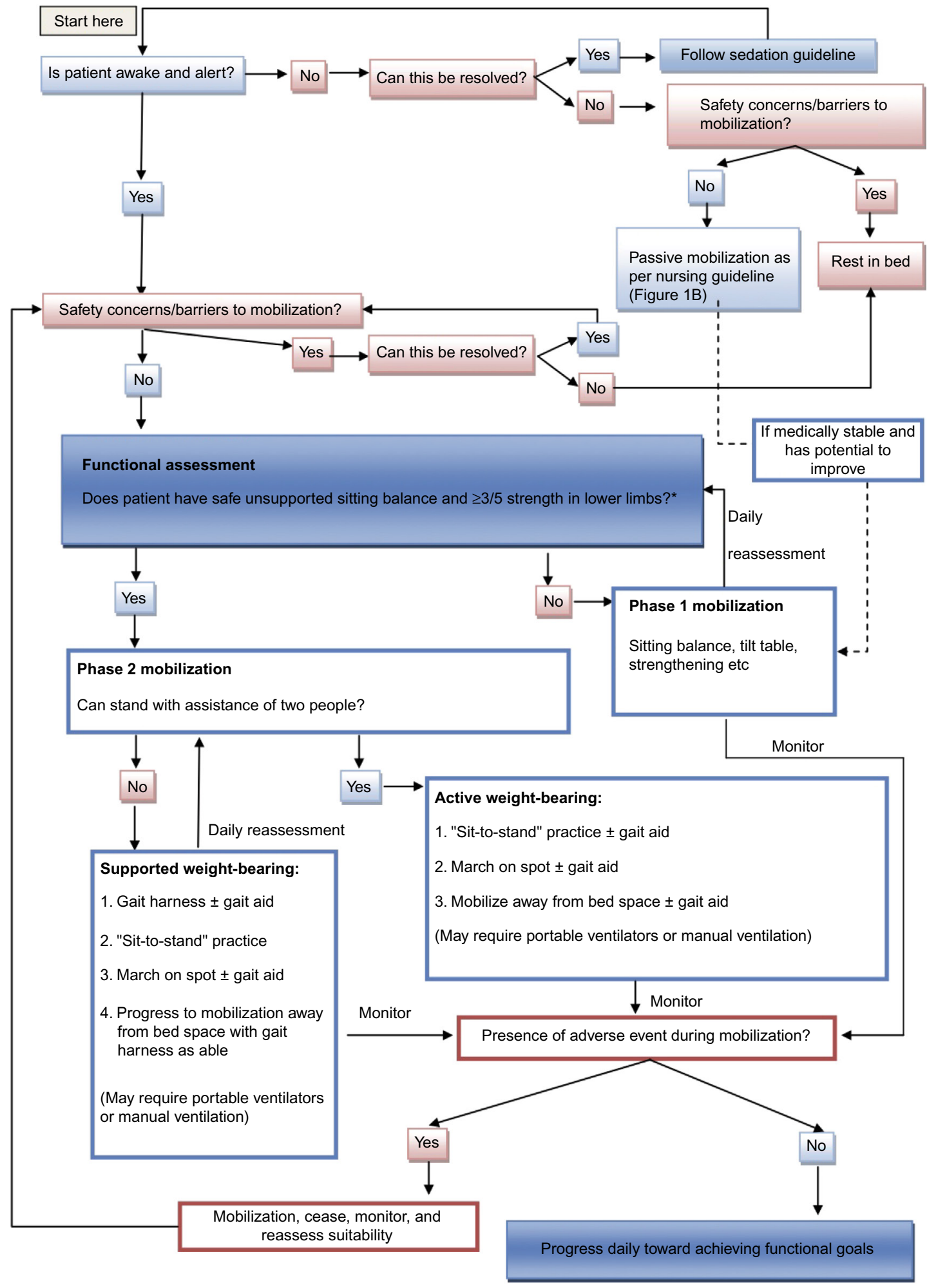

Figure I (Continued). 
- Passive mobilization (hoist transfer to sit-out-of-bed)

- Staff: minimum of two to three (bedside nurse \pm support staff), one staff member designated to airway, ensure safety of attachments

- Physiotherapy input not required to activate this step

- Phase 2 mobilization: active weightbearing (eg, sit-to-stand, stand transfer to sit-out-of-bed)

- Initial mobilization episodes require physiotherapy input

- Staff: two to three (if more staff required, physiotherapy input recommended), one staff member designated to airway, ensure safety of attachments

- May require a gait aid (eg, forearm support frame)

- Mobilization away from the bedspace requires physiotherapy input

\section{Mobilization of spontaneously ventilating patients}

- Passive mobilization (hoist transfer to sit-out-of-bed)

- Staff: minimum of two (bedside nurse \pm support staff), ensure safety of attachments

- Physiotherapy input not required to activate this step

- Phase 2 mobilization: active weightbearing (eg, sit-to-stand, stand transfer to sit-out-of-bed)

- Staff: one to two (eg, nurse \pm support staff), ensure safety of attachments

- May require a gait aid (eg, forearm support frame)

- Physiotherapy consultation if concerned or unclear of mobility recommendations

- If mobilization away from the bedspace requires more than one staff member, recommend physiotherapy input

Figure I Mobilization flowchart.

Notes: (A) Decision making flowchart for mobilization of ICU patients. *If hemiplegic, $\geq 3 / 5$ on intact side. (B) Nursing guideline for mobilization of ICU patients. Abbreviation: ICU, intensive care unit.

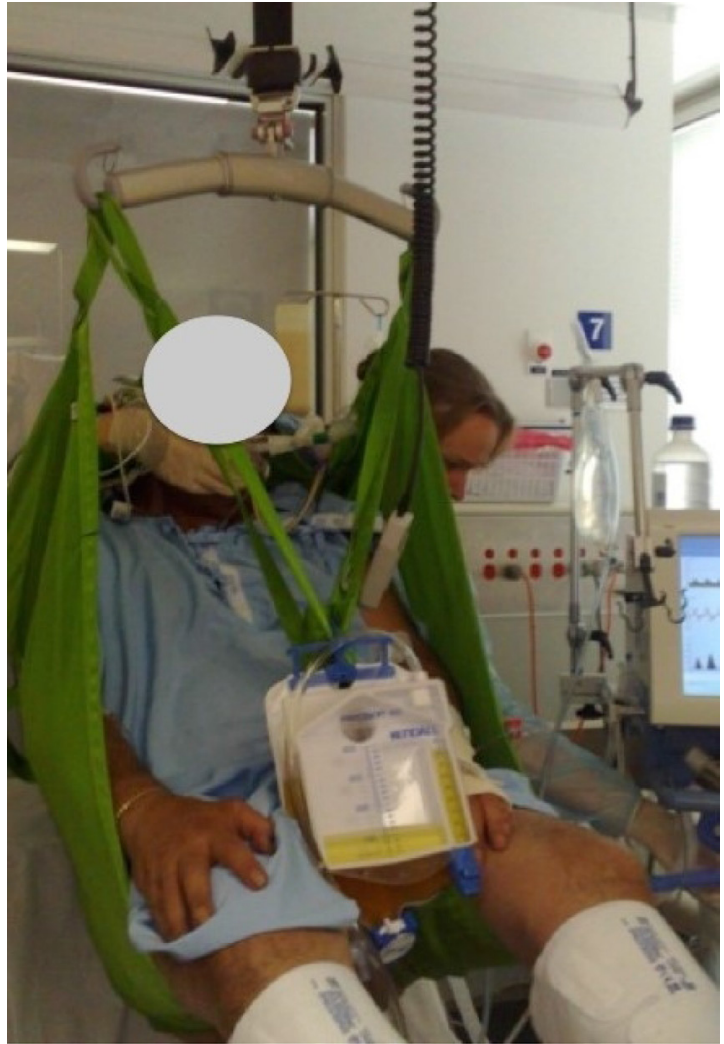

Figure 2 Passive mobilization.

Notes: Passive mobilization - hoist transfer from bed to chair for an unresponsive intubated patient. Note the support of the airway and organization of lines. sitting position in an appropriate chair to potentially minimize orthostatic intolerance, which is known to occur after just 24 hours of bed rest. ${ }^{21}$ A portable sling lifter for mobilization is feasible and is a standard practice in our unit.

\section{Active mobilization}

Once the decision to actively mobilize a patient is made, the next step requires the physiotherapist to complete a functional assessment, including a sitting balance and strength assessment. If the patient does not have at least 3/5 (Oxford scale) strength in their lower limbs or safe unsupported sitting balance, the patient commences "phase 1 mobilization" (Figure 1). Phase 1 can involve sitting balance retraining (eg, reaching and returning to midline from the bed or chair), strength training including the use of weights or slings, and/ or treatment on the tilt table (Figure 3). Some patients in the passive mobilization group (eg, patients with a neurological diagnosis) may be assessed as suitable for "phase 1 mobilization" even if they are unable to participate in therapy or their level of consciousness fluctuates. A patient will remain in this phase until they achieve adequate sitting balance and lower limb strength to progress to "phase 2 mobilization".

If the patient is not able to stand with the assistance of two staff, they progress to the "supported weight-bearing" 
A

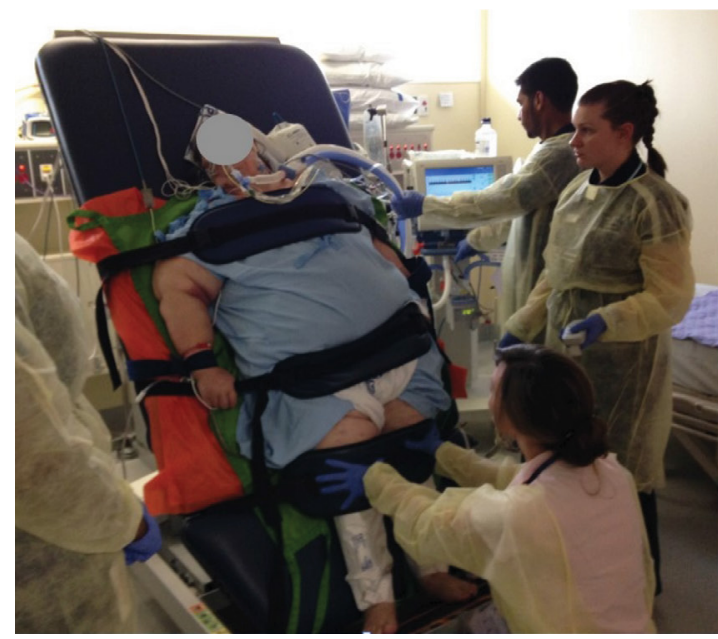

B

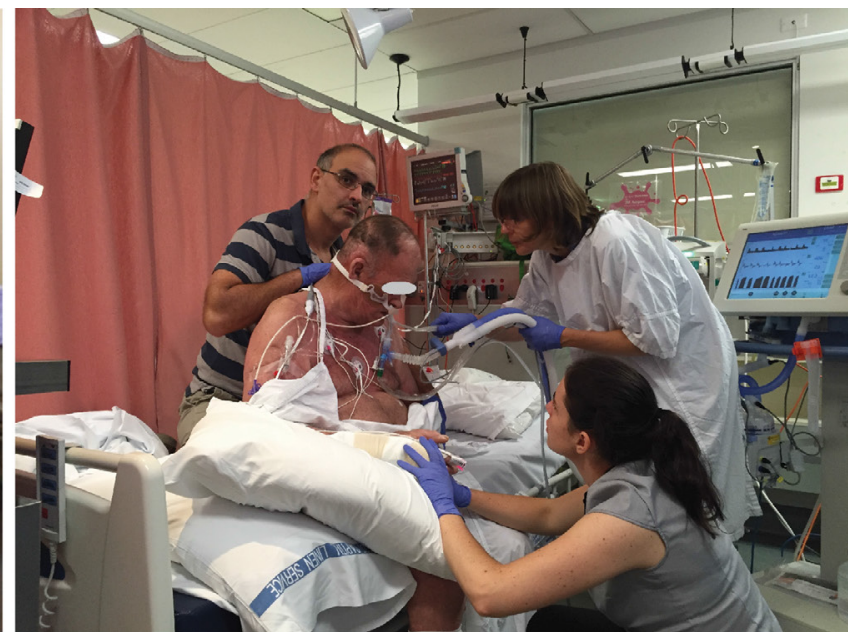

Figure 3 Phase I mobilization.

Notes: (A) Tilt table for an awake intubated patient performing squats with assistance. This patient was unable to achieve supported weight-bearing with a gait harness. Note the nurse managing airway and physiotherapists providing support to knees. (B) Sitting balance with a ventilator-dependent patient. Note the nurse providing airway support while physiotherapists assist balance behind and in front of the patient.

phase, which involves the use of a gait harness to facilitate mobilization (Figure 4). A gait harness can be suspended from overhead ceiling tracking or from a sling hoist lifter. For patients with several drains and attachments in the abdomen and thorax, we recommend noncircumferential gait harnesses (Figure 4) as these are less likely to result in compression or dislodgement of these items. The sling is ideally placed under the patient's pelvis from a seated position. This sometimes requires the patient to be lifted with a standard sling, and then lowered onto the gait harness either on the edge of the bed or in a chair. We also recommend padding the gait harness with a disposable lining to reduce the need for laundering between treatments. Once transferred to a standing position, the patient can be assisted to extend the hips and knees as much as possible and step forward, or practice lateral weight shift.

If the patient is able to stand with the assistance of two staff members, they proceed to the "active weight-bearing phase" (Figure 5). Patients may require the use of a gait aid (eg, a forearm support frame), and some patients will require a sling hoist to sit out of bed, with more effective mobilization commencing from the chair. Mobilization is progressive both within and between treatments to achieve functional goals (Figure 1, phase 2 mobilization).

For some chronically critically ill patients, following discussion and planning with the MDT, it has been possible to complete rehabilitation sessions outside of the bed space, including the balcony area adjacent to our ICU and the rehabilitation gym. If rehabilitation is occurring outside the ICU location, we ensure an airway management kit is

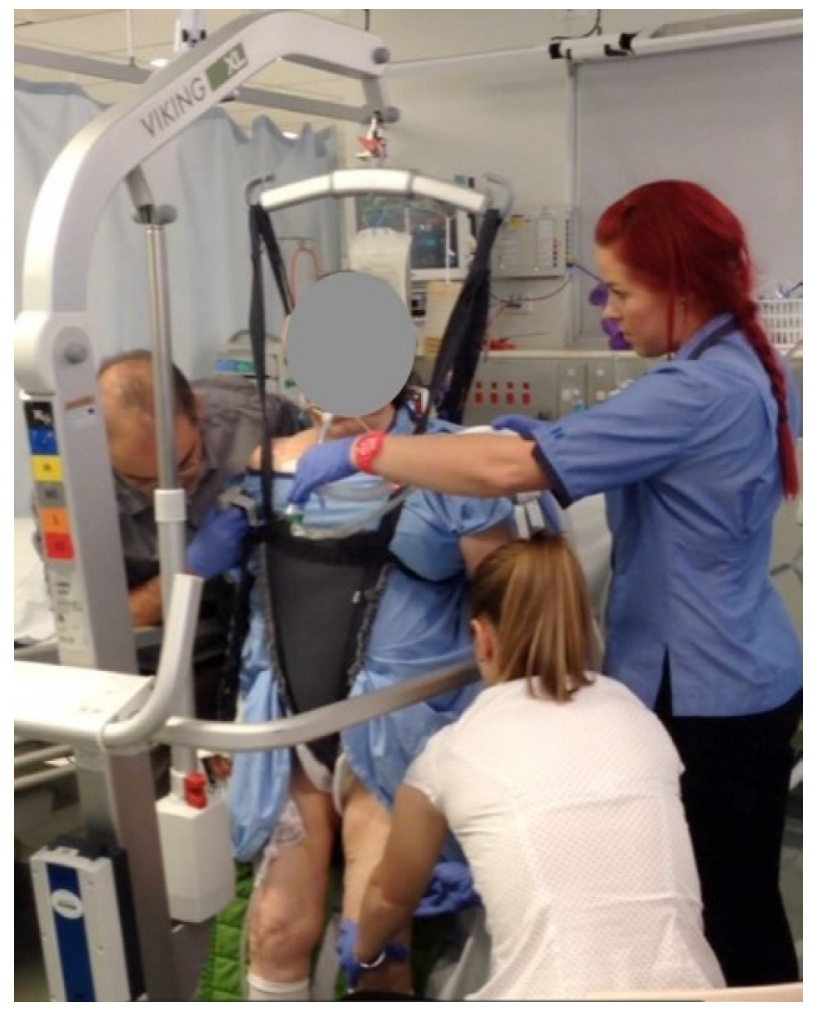

Figure 4 Phase 2 mobilization: supported weight-bearing for an intubated patient with primary central nervous system pathology.

Notes: Note the gait harness providing body weight support through the pelvis and physiotherapists assisting with hip and knee control while the nurse supports the airway.

with the patient at all times, as well as nursing and medical escorts as appropriate. For example, we have had a mechanically ventilated patient complete a 30-minute therapy session in the rehabilitation gym, including treadmill training on a portable ventilator. 
A

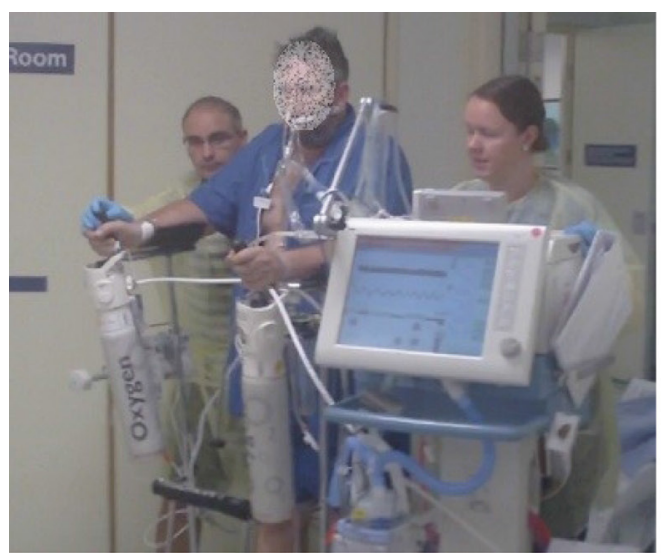

B

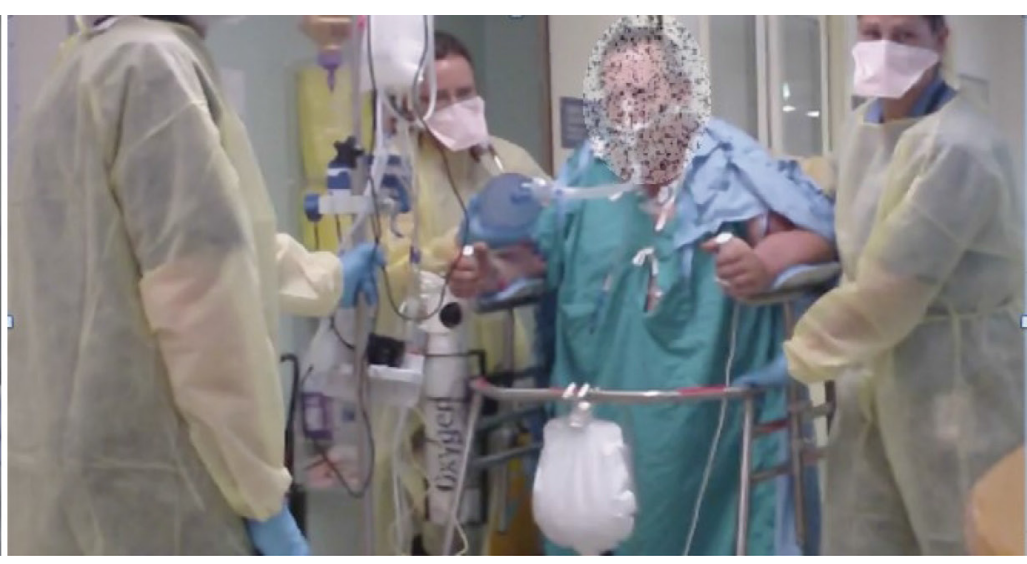

Figure 5 Phase 2 mobilization: active mobilization of awake intubated patients.

Notes: (A) With a ventilator and portable monitoring. Note the spare oxygen cylinders suspended from the forearm support frame. Support staff are following the patient with a chair. (B) With resuscitator bag. Note the bariatric forearm support frame and organization of attachments on the portable pole. Both the physiotherapist and nurse are assisting the patient to steer the frame.

\begin{tabular}{|c|c|}
\hline Preparation & $\begin{array}{l}\text { - Case discussion with the MDT } \\
\text { - Patient aware of the plan before commencing mobilization } \\
\text { - Equipment (check safe weight limit) } \\
\text { - Physical environment }\end{array}$ \\
\hline Leader & - Clear communication and designation of roles within the team \\
\hline Airway and emergency equipment & $\begin{array}{l}\text { - Designated staff member to the role of "airway" } \\
\text { - Airway appropriately secured and grade of intubation noted } \\
\text { - All emergency equipment present } \\
\text { - Airway clearance }\end{array}$ \\
\hline Number of staff & $\begin{array}{l}\text { - Number of staff from the MDT available with required manual handling } \\
\text { and clincial skills }\end{array}$ \\
\hline Backup plan & $\begin{array}{l}\text { - Discussion with the patient and the MDT regarding the plan } \\
\text { - Equipment available }\end{array}$ \\
\hline
\end{tabular}

Figure 6 Plan B mnemonic for mobilization of intubated patients.

Abbreviation: MDT, multidisciplinary team.

\section{Mobilizing an invasively mechanically ventilated patient}

Invasive mechanical ventilation, whether through an endotracheal or tracheostomy tube, should not be a barrier for mobilization and has been shown to be safe and feasible. ${ }^{8-10}$ Considerable planning and preparation is required to ensure safety for both patient and staff. We have developed the "Plan B" mnemonic (Figure 6) which is used routinely by staff in our unit before commencing mobilization of a mechanically ventilated patient.

\section{P - preparation}

Preparation and planning are essential, as mobilization of an ICU patient requires coordination of the patient, several staff, and equipment. Timing of the mobilization episode needs to be negotiated with the bedside nurse and coordinated with other care needs (eg, procedures and scans). In our experience, $30-60$ minutes is a reasonable time frame to allocate for mobilization of a ventilated patient, including preparation time. Mobilization should be timed to coincide with medication peak effectiveness where applicable (eg, 
analgesia and bronchodilators). Equipment requirements may include portable monitoring, overhead ceiling-mounted tracking or portable sling lifters, gait harnesses, tilt table, gait aid (eg, forearm support frame), a suitable recliner chair, and linen. If mobilizing away from the bed space, other equipment may include portable oxygen, portable suction, and either a portable ventilator or resuscitator bag. When using a portable ventilator, a spare oxygen cylinder should accompany the patient to guarantee adequate oxygen supply. The safe weight limit of all equipment should be checked with the patient's body weight. When using gait harnesses, patency of all lines and attachments should be checked prior to proceeding with and at multiple time points during mobilization.

Preparation of the awake ventilated patient should include provision of a clear explanation of the plan, process, goals, and rationale for mobilization, answering questions, and providing reassurance as required.

A final review of the physical environment (eg, the bed space) completes the preparation phase. This must include a consideration of lines and attachments to ensure that there is adequate space to perform mobilization safely. If lines are to be removed imminently, consider delaying mobilization until after this has occurred. Often, it is possible to rationalize attachments to reduce the number of staff required for safe mobilization. It is highly recommended to remove any unnecessary equipment and clutter out of the area.

\section{$\mathrm{L}$ - leader}

Throughout the mobilization episode, it is imperative that there is a clear leader. In our unit, this is usually the physiotherapist, coordinating between the patient, the bedside nurse, support staff, and sometimes, family members as well. Members of the mobilization team must have a clear understanding of their role (eg, airway, attachments, patient, and frame) with clear communication throughout the treatment. However, while the physiotherapist may typically lead the mobilization, the person responsible for the airway reserves the right to revert to a leadership role at any point, as airway safety must be the first priority. In this case, in our unit, the physiotherapist would temporarily transfer leadership to the bedside nurse who is usually responsible for the airway. These leadership transfers are articulated clearly so that the whole team is aware of the change to ensure patient safety.

\section{A - airway and emergency equipment}

All mobilization episodes should aim to minimize patient risk. Emergency equipment including emergency airway and ventilation equipment should be checked as part of routine nursing practices. Discussion with the ICU medical team prior to mobilization is recommended for any patient with an identified "difficult airway" (eg, documented grade 3 or 4 Modified Cormack-Lehane score at the time of intubation) or if there is any concern regarding airway stability. Prior to mobilization, all artificial airways should be reviewed and appropriately secured. One staff member (usually the bedside nurse) is dedicated to the role of "airway" and is responsible for supporting the airway to prevent tube migration or displacement. For patients with a high sputum load, airway clearance is recommended prior to any mobilization. If mobilizing a patient with a high sputum load away from the bed space, portable suction must be available.

\section{$\mathrm{N}$ - number of staff}

The number of staff required to safely mobilize a critically ill patient is risk-assessed on the basis of the patient's functional assessment. The minimum number of staff required to mobilize a ventilated patient in our ICU is two (one bedside nurse for the airway and one physiotherapist for the patient), but this is only possible where the patient requires minimal physical support. More commonly, we require between three and five staff members to mobilize a ventilated patient and safely manage all the lines and attachments. Our ICU staffing includes dedicated support staff who are available to assist with mobilization. Alternatives could include other nursing or physiotherapy staff or allied health assistants. It is essential that the MDT members are trained in safe manual handling and have a clear understanding of each other's roles.

\section{B - backup plan}

Before mobilization, the leader should clearly articulate the backup plan, which may occur if the patient is unable to complete the planned mobilization episode. The patient should also be aware of the backup plan, so that they are aware of the possible change in direction midtreatment. Examples of this include having the bed nearby, a chair, commode, or wheelchair to follow the patient if mobilizing away from the bed space or a gait harness to prevent a fall in a high-risk patient. Sometimes the backup plan is simply to sit the patient back in the chair if the patient has insufficient standing ability to proceed.

\section{Discussion}

We have described strategies to guide the practicalities of safe early mobilization of ICU patients, including those who 
are unable to actively participate. This approach has been used successfully in our ICU for more than 10 years with a very low incidence of adverse outcomes, ie, $1.1 \% .{ }^{14}$ The two "adverse outcomes" in our audit were both instances of hypotension requiring intervention (ie, return to bed, fluid loading, and transient increase in vasopressor requirements) with no long-term consequences. This is consistent with other available safety data, including a recent review which reported an incidence of $\leq 4 \%$ adverse events with early mobilization in ICU ${ }^{22}$ and a study of 5,267 ICU physical therapy sessions where a physiological abnormality or a potential safety event occurred in $34(0.6 \%)$ sessions. $^{23}$

Progressive mobilization protocols have been published from the United States ${ }^{3,12,24}$ and the United Kingdom. ${ }^{11}$ Bassett et $\mathrm{al}^{12}$ published a goal-directed progressive mobility continuum including safety criteria. All these protocols involve the initial step of passive range of motion exercises. Our clinical practice differs as no member of our MDT routinely performs passive range of motion exercises given the absence of evidence that these reduce or prevent contractures in critically ill patients. ${ }^{25,26}$ The absence of passive mobilization is consistent with surveyed Australian intensive care physiotherapy practice. ${ }^{27}$ Another important distinction from other protocols is that we aim to sit our patients out of bed for a minimum of 4 hours per day with pressure care performed in the chair. Although Bassett et al ${ }^{12}$ targets a frequency of mobilization at two to three times per day, we have opted to individualize the frequency of mobilization. Many of our patients are unlikely to tolerate this frequency due to fatigue. Fatigue was a limiting factor in a recent randomized controlled trial of a protocolized rehabilitation program commencing in ICU, where exercise prescription was targeted to be delivered twice per day but was only feasible in $55 \%$ of potential sessions. $^{28}$

Despite the evidence in favor of early mobilization, substantial barriers exist in many ICUs, including our own. In 2008, we audited our mobilization practice and found that patients were mobilized on $54 \%$ of patient days, with avoidable factors identified in $47 \%$ of cases where patients were not mobilized. ${ }^{14}$ Specifically, the most common perceived barriers were femoral vascular access, (particularly femoral dialysis catheters), timing of procedures, and patient agitation or low level of consciousness. More recent evidence indicates that femoral access should not prohibit early mobilization, ${ }^{29}$ and, in the case of dialysis catheters, mobilization may actually prolong filter life. ${ }^{30}$ Other potential barriers to mobilization may include staffing, equipment, leadership, referral processes, delirium, sedation, and perceived lack of safety. Strategies to overcome these barriers have been published to assist clinicians in increasing mobility in ICU. ${ }^{10,31}$

Our ICU sedation practices are goal-directed to achieve a target Richmond Agitation-Sedation Score.${ }^{18}$ For patients in whom deep sedation is not indicated, sedation is targeted to achieve a Richmond Agitation-Sedation Score of -1 to 1 , as opposed to daily sedation interruption. Oversedation may be a barrier to mobilization, may contribute to delirium, and compromise the patient's ability to participate in mobilization. Review of sedation practices may be a key step in increasing mobilization in critically ill patients. A strategy to reduce heavy sedation and increase mobilization in medical ICU patients has been shown to be safe, reduce delirium, improve function, and reduce ICU and hospital length of stay. ${ }^{1}$ The optimization of sedation to facilitate mobilization clearly requires close multidisciplinary collaboration between medical, nursing, and physiotherapy staff. In our ICU, this interaction and negotiation occurs frequently not just in handover meetings and ward rounds, but ad hoc across the day as issues arise. This open multidisciplinary communication requires mutual respect for each other's roles and expertise, and an understanding of the priorities of competing demands for care of acutely unwell patients.

The description of our experience and approach to early mobilization in ICU adds to the body of evidence by providing specific practical details of how to perform this safe and feasible intervention as a MDT. A limitation of the material presented in this paper is that the ideal timing, type, frequency, intensity, and duration of mobilization required to prevent or minimize functional impairments in ICU survivors are yet to be established. Our approach is a feasible bundle of care that promotes early mobilization in critically ill patients; however, the effect of the individual components cannot be currently demonstrated. Given the heterogeneity of general ICU presentations, individualized goal-directed prescription is warranted. Clinicians need to make informed patient-centered decisions balancing the risks of prolonged bed rest against the benefits of early mobilization. Due to the multidisciplinary nature of the ICU environment, the decision to mobilize an ICU patient should be shared between the physiotherapy, medical, and nursing staff.

Developing and sustaining an ICU MDT culture that promotes, values, and prioritizes early mobilization is essential to translating evidence into clinical practice. Numerous articles are available to assist in units developing an early 
mobilization culture. ${ }^{1,10,12,16,32,33}$ In our experience, early mobilization is both feasible and safe and is the result of a concerted commitment from the MDT to make early mobilization the norm, rather than the exception. It is also possible that, despite the contrast of our approach with other published guidelines, we are still too conservative, and the limits of early proactive rehabilitation in ICU are yet to be established.

\section{Conclusion}

Early progressive mobilization is safe and feasible in critically ill patients and requires close collaboration of the MDT on a daily basis. It is our hope that the guided clinical reasoning and practical considerations described in this paper will provide tools that allow frontline clinical staff to implement early mobilization in the majority of critically ill patients in a safe and effective manner.

\section{Acknowledgments}

The authors gratefully acknowledge the staff of the Physiotherapy Department and Intensive Care Unit of Canberra Hospital for their support in developing this protocol. They also gratefully acknowledge the patients, students, and staff who each kindly consented to publication of the photographs in this report. They thank the Canberra Hospital ICU Research Executive Committee and the ACT Health Chief Allied Health Office for their financial support of this publication.

\section{Disclosure}

The authors report no conflicts of interest in this work.

\section{References}

1. Needham DM, Korupolu R, Zanni JM, et al. Early physical medicine and rehabilitation for patients with acute respiratory failure: a quality improvement project. Arch Phys Med Rehabil. 2010;91(4):536-542.

2. Bailey P, Thomsen GE, Spuhler VJ, et al. Early activity is feasible and safe in respiratory failure patients. Crit Care Med. 2007;35(1):139-145.

3. Morris PE, Goad A, Thompson C, et al. Early intensive care unit mobility therapy in the treatment of acute respiratory failure. Crit Care Med. 2008;36(8):2238-2243.

4. Schweickert WD, Pohlman MC, Pohlman AS, et al. Early physical and occupational therapy in mechanically ventilated, critically ill patients: a randomised controlled trial. Lancet. 2009;373(9678): 1874-1882.

5. Kayambu G, Boots R, Paratz J. Physical therapy for the critically ill in the ICU: a systematic review and meta-analysis. Crit Care Med. 2013;41(6):1543-1554.

6. Stiller K. Physiotherapy in intensive care: an updated systematic review. Chest. 2013;144(3):825-847.

7. Morris PE, Griffin L, Berry M, et al. Receiving early mobility during an intensive care unit admission is a predictor of improved outcomes in acute respiratory failure. Am J Med Sci. 2011;341(5):373-377.

8. Nydahl P, Ruhl AP, Bartoszek G, et al. Early mobilization of mechanically ventilated patients: a 1-day point-prevalence study in Germany. Crit Care Med. 2014;42(5):1178-1186.
9. Berney SC, Harrold M, Webb SA, et al. Intensive care unit mobility practices in Australia and New Zealand: a point prevalence study. Crit Care Resusc. 2013;15(4):260-265.

10. Engel HJ, Needham DM, Morris PE, Gropper MA. ICU early mobilization: from recommendation to implementation at three medical centers. Crit Care Med. 2013;41(9 Suppl 1):S69-S80.

11. McWilliams D, Weblin J, Atkins G, et al. Enhancing rehabilitation of mechanically ventilated patients in the intensive care unit: a quality improvement project. J Crit Care. 2015;30(1):13-18.

12. Bassett RD, Vollman KM, Brandwene L, Murray T. Integrating a multidisciplinary mobility programme into intensive care practice (IMMPTP): a multicentre collaborative. Intensive Crit Care Nurs. 2012;28(2):88-97.

13. Hodgson CL, Stiller K, Needham DM, et al. Expert consensus and recommendations on safety criteria for active mobilization of mechanically ventilated critically ill adults. Crit Care. 2014;18(6):658.

14. Leditschke IA, Green M, Irvine J, Bissett B, Mitchell IA. What are the barriers to mobilizing intensive care patients? Cardiopulm Phys Ther J. 2012;23(1):26-29.

15. Stiller K. Safety issues that should be considered when mobilizing critically ill patients. Crit Care Clin. 2007;23(1):35-53.

16. Gosselink R, Bott J, Johnson M, et al. Physiotherapy for adult patients with critical illness: recommendations of the European Respiratory Society and European Society of Intensive Care Medicine Task Force on Physiotherapy for Critically Ill Patients. Intensive Care Med. 2008;34(7): $1188-1199$.

17. Hanekom S, Berney S, Morrow B, et al. The validation of a clinical algorithm for the prevention and management of pulmonary dysfunction in intubated adults - a synthesis of evidence and expert opinion. J Eval Clin Pract. 2011;17(4):801-810.

18. Sessler CN, Gosnell MS, Grap MJ, et al. The Richmond AgitationSedation Scale: validity and reliability in adult intensive care unit patients. Am J Respir Crit Care Med. 2002;166(10):1338-1344.

19. Borg G. Ratings of perceived exertion and heart rates during short-term cycle exercise and their use in a new cycling strength test. Int $J$ Sports Med. 1982;3(3):153-158.

20. Powers J, Bennett SJ. Measurement of dyspnea in patients treated with mechanical ventilation. Am J Crit Care. 1999;8(4):254-261.

21. Khan MH, Kunselman AR, Leuenberger UA, et al. Attenuated sympathetic nerve responses after 24 hours of bed rest. Am J Physiol Heart Circ Physiol. 2002;282(6):H2210-H2215.

22. Adler J, Malone D. Early mobilization in the intensive care unit: a systematic review. Cardiopulm Phys Ther J. 2012;23(1):5-13.

23. Sricharoenchai T, Parker AM, Zanni JM, Nelliot A, Dinglas VD, Needham DM. Safety of physical therapy interventions in critically ill patients: a single-center prospective evaluation of 1110 intensive care unit admissions. J Crit Care. 2014;29(3):395-400.

24. Engel HJ, Tatebe S, Alonzo PB, Mustille RL, Rivera MJ. Physical therapist-established intensive care unit early mobilization program: quality improvement project for critical care at the University of California San Francisco Medical Center. Phys Ther. 2013;93(7):975-985.

25. Stockley RC, Morrison J, Rooney J, Hughes J. Move it or lose it? A survey of the aims of treatment when using passive movements in intensive care. Intensive Crit Care Nurs. 2012;28(2):82-87.

26. Prabhu RK, Swaminathan N, Harvey LA. Passive movements for the treatment and prevention of contractures. Cochrane Database Syst Rev. 2013;12:CD009331.

27. Wiles L, Stiller K. Passive limb movements for patients in an intensive care unit: a survey of physiotherapy practice in Australia. J Crit Care. 2010;25(3):501-508.

28. Berney S, Haines K, Skinner EH, Denehy L. Safety and feasibility of an exercise prescription approach to rehabilitation across the continuum of care for survivors of critical illness. Phys Ther. 2012;92(12): 1524-1535.

29. Damluji A, Zanni JM, Mantheiy E, Colantuoni E, Kho ME, Needham DM. Safety and feasibility of femoral catheters during physical rehabilitation in the intensive care unit. J Crit Care. 2013;28(4):535.e9-e15. 
30. Wang YT, Haines TP, Ritchie P, et al. Early mobilization on continuous renal replacement therapy is safe and may improve filter life. Crit Care. 2014;18(4):R161.

31. Lipshutz AKM, Engel H, Thornton K, Gropper MA. Early mobilization in the intensive care unit: evidence and implementation. ICU Director. 2012;3(10):10-16.
32. Bailey PP, Miller RR 3rd, Clemmer TP. Culture of early mobility in mechanically ventilated patients. Crit Care Med. 2009;37(10 Suppl):S429-S435.

33. Dafoe S, Chapman MJ, Edwards S, Stiller K. Overcoming barriers to the mobilisation of patients in an intensive care unit. Anaesth Intensive Care. 2015;43(6):719-727.

\section{Publish your work in this journal}

The Journal of Multidisciplinary Healthcare is an international, peerreviewed open-access journal that aims to represent and publish research in healthcare areas delivered by practitioners of different disciplines. This includes studies and reviews conducted by multidisciplinary teams as well as research which evaluates the results or conduct of such teams or health- care processes in general. The journal covers a very wide range of areas and welcomes submissions from practitioners at all levels, from all over the world. The manuscript management system is completely online and includes a very quick and fair peer-review system. Visit http://www.dovepress.com/ testimonials.php to read real quotes from published authors.

Submit your manuscript here: https://www.dovepress.com/journal-of-multidisciplinary-healthcare-journal 